\title{
Competition and stoichiometry: coexistence of two predators on one prey
}

\author{
Irakli Loladze, ${ }^{\text {a,* }}$ Yang Kuang, ${ }^{\mathrm{b}}$ James J. Elser, ${ }^{\mathrm{c}}$ and William F. Fagan ${ }^{\mathrm{d}}$ \\ ${ }^{a}$ Department of Ecology \& Evolutionary Biology, Princeton University, Princeton, NJ 08544-1003, USA \\ ${ }^{\mathrm{b}}$ Department of Mathematics, Arizona State University, Tempe, AZ 85287-1804, USA \\ ${ }^{\mathrm{c}}$ Department of Biology, Arizona State University, Tempe, AZ 85287-1501, USA \\ ${ }^{\mathrm{d}}$ Department of Biology, University of Maryland, College Park, MD 20742, USA
}

Received 14 October 2001

\begin{abstract}
The competitive exclusion principle (CEP) states that no equilibrium is possible if $n$ species exploit fewer than $n$ resources. This principle does not appear to hold in nature, where high biodiversity is commonly observed, even in seemingly homogenous habitats. Although various mechanisms, such as spatial heterogeneity or chaotic fluctuations, have been proposed to explain this coexistence, none of them invalidates this principle. Here we evaluate whether principles of ecological stoichiometry can contribute to the stable maintenance of biodiverse communities. Stoichiometric analysis recognizes that each organism is a mixture of multiple chemical elements such as carbon $(\mathrm{C})$, nitrogen $(\mathrm{N})$, and phosphorus $(\mathrm{P})$ that are present in various proportions in organisms. We incorporate these principles into a standard predator-prey model to analyze competition between two predators on one autotrophic prey. The model tracks two essential elements, $\mathrm{C}$ and $\mathrm{P}$, in each species. We show that a stable equilibrium is possible with two predators on this single prey. At this equilibrium both predators can be limited by the $\mathrm{P}$ content of the prey. The analysis suggests that chemical heterogeneity within and among species provides new mechanisms that can support species coexistence and that may be important in maintaining biodiversity.
\end{abstract}

(C) 2003 Elsevier Inc. All rights reserved.

Keywords: Competition; Stoichiometry; Food quality; C:P ratio; Consumer-resource

\section{Introduction}

One of the oldest and most intriguing paradigms in community ecology - the competitive exclusion principle (CEP) - states that at most $n$ species can coexist on $n$ resources (Volterra, 1926; Gause, 1934; Hardin, 1960; MacArthur and Levins, 1964; Levin, 1970). The mathematical validity of this principle is largely in disagreement with observations of a vast diversity of species supported by seemingly few resources. The renewed interest in this principle is fueled by its relevance to a central problem in modern ecology: finding and understanding mechanisms that maintain the earth's vast biodiversity. Since the time Hardin $(1959,1960)$ coined the term "the competitive exclusion

\footnotetext{
${ }^{*}$ Corresponding author. Fax: + 1-413-215-7100.

E-mail addresses: iloladze@princeton.edu (I. Loladze), kuang@asu.edu (Y. Kuang), j.elser@asu.edu (J.J. Elser), bfagan@glue.umd.edu (W.F. Fagan).
}

principle," subsequent advances in theoretical ecology (by no means a complete list below) have identified several mechanisms that promote diversity. First, if resources are not explicitly modeled, competitive exclusion is not mandatory: Strobeck (1973) showed that $n$ species can coexist at a locally stable equilibrium in a homogeneous (spatially and temporally) classical Lotka-Volterra model with no resources. In such a model, however, competition is expressed in an ad hoc fashion via constant competition coefficients. In explicit resource models, exploitative competition among consumers is realized, as in real life, directly via the consumption of shared resources. Second, even for models that explicitly consider resources, the coexistence of $n$ species on fewer than $n$ resources is possible via internally produced limit cycles (Armstrong and McGehee, 1976; Abrams and Holt, 2002) or chaotic fluctuations (Armstrong and McGehee, 1980; Huisman and Weissing, 1999, 2000). Third, other factors such as spatial (Durrett and Levin, 1994; Richards et al., 2000) or temporal (Butler et al., 
1985; Lenas and Pavlou 1995; Chesson, 1994) heterogeneity, light fluctuations (Litchman and Klausmeier, 2001), predation (Holt and Lawton, 1994; Leibold, 1996; Chase et al., 2002), disturbance (Hastings, 1980), and interference between consumers (Vance, 1984, 1985; Kuang et al., 2003) provide mechanisms for coexistence.

Considering the multitude of various definitions of CEP in the literature, for the sake of clarity, here by the CEP, we mean the following concise formulation: a stable equilibrium is impossible in a homogeneous system with $n$ species exploiting fewer than $n$ resources. Note that exploitative competition is assumed here; that is, a situation in which consumers do not interfere directly but affect each other via the consumption of shared resources. In addition, spatial and temporal homogeneity is assumed. Within these restrictions, however, the CEP is extremely robust to various types of models: whether resources are abiotic such as chemical elements or biotic such as prey species (Armstrong and McGehee, 1980), essential or substitutable (Leon and Tumpson, 1975; Tilman, 1982; Grover, 1997) the CEP holds firmly. All these models share the same general structure: one set of equations describes resource dynamics and the second set describes the dynamics of consumers. The CEP follows as a rigorous mathematical result under an assumption that consumers and resources are in a predator-prey relationship. In other words, the partial derivative of consumer abundance with respect to resource abundance is nonnegative, while the partial derivative of resource abundance with respect to the consumer is nonpositive. This assumption seems to be so commonsense that it is usually incorporated into models without questioning its validity. We will show, however, that in our model mass balance laws and stoichiometric properties can break this rule, that is, higher resource abundance can hurt consumer growth, which in turn can facilitate coexistence of competing consumers.

In particular, any biotic resource (hereafter, prey), as all life, consists of multiple abiotic resources that are essential to its consumers. All known organisms require carbon $(\mathrm{C})$, oxygen $(\mathrm{O})$, hydrogen $(\mathrm{H})$, nitrogen $(\mathrm{N})$, and phosphorus $(\mathrm{P})$ (in addition, all cellular organisms require sulfur $(\mathrm{S})$, potassium $(\mathrm{K})$, calcium $(\mathrm{Ca})$, sodium $(\mathrm{Na})$, and other elements). Moreover, while consumer species share the requirement for the same nutrients, they can differ in their chemical composition. In addition to this chemical variety among consumer species, autotrophic resources (i.e. plants and algae) are known to exhibit highly variable intraspecific chemical composition (Sterner and Elser, 2002). Can such "chemical diversity" within and among species enhance biodiversity via previously unrecognized mechanisms? Answering this complex question is outside of the scope of this paper and our general abilities, but within the CEP framework, we can attack a closely related but simpler question: can several consumers stably coexist one a single prey that itself consists of multiple chemical elements? The idea that a single prey can represent more than one resource is not novel. For example, different plant parts can provide distinct resources to herbivores (Tilman, 1982). Abrams (1988) demonstrated that variation in coloration among individuals of a single prey species can provide more than one potential resource to consumers. What is novel in our approach is its reliance not on a particular species-specific properties but on an undisputable fact that any prey is always a package of multiple chemical elements to all of its consumers.

Our choice of chemical elements rather than complex biochemicals as a preferred "chemical scale" for modeling is not accidental. The overwhelming complexity of organic compounds and their intricate metabolic pathways leave us no hope to follow them with a tractable model. However, on a finer scale of chemical elements a simple yet powerful mass conservation law holds with respect to each chemical element. Alfred Lotka (1925), a physical chemist and an originator of the famous Lotka-Volterra equations, advocated the use of chemical elements as an essential tool for understanding biological dynamics. Most recently, Sterner and Elser (2002) have expanded Lotka's vision to develop the field of ecological stoichiometry.

Predator-prey and competitive interactions are among the major forces that shape food webs and ecological communities. It has been shown both experimentally (Urabe and Sterner, 1996; Sterner et al., 1998; Nelson et al., 2001; Urabe et al., 2002, 2003) and theoretically (Andersen, 1997; Loladze et al., 2000; Muller et al., 2001), that variable chemical composition (i.e. stoichiometry) of prey can significantly affect predator-prey dynamics. We are not aware, however, of any prior work that addresses the issue of variable prey stoichiometry and competition among herbivores.

Here, by capturing the critical elements of ecological stoichiometry, we construct the simplest, in our opinion, model needed to address this issue. Our motivation is to find out whether it is at all possible for two herbivores to stably coexist on one prey of variable quality. In the next section, we construct the model. In Section 3, we analyze it qualitatively, and then numerically and graphically (with Monod functional responses). We also construct bifurcation diagrams of the system along an implicit light gradient. In the discussion, we address the implications of these findings and suggest further directions.

\section{Model}

We model two consumers exploiting one prey in a system with no spatial heterogeneity or external 
variability. Our approach differs from conventional consumer-resource models, because we do not assume, a priori, that higher prey density must either increase predator growth or, in the case of feeding saturation, leave it unchanged. Instead, the law of mass conservation will determine consumer growth in our model.

To have some concrete system in mind, we can think of the prey as a single species of alga, while the consumers are two distinct zooplankton species, all placed in a well-mixed system open only to light and air. (One can imagine a continuously stirred culture in a uniformly lighted chamber with open top and clear walls.) In the model construction, we follow a course outlined in Loladze et al. (2000), which deals with one consumer-one prey interactions.

Let us start with a conventional (MacArthurRosenzweig-type) model, which describes a system of two consumers feeding on one prey, here assumed to be a photoautotroph at the base of the food web (e.g. phytoplanktonic algae):

$\frac{d x}{d t}=r x\left(1-\frac{x}{K}\right)-f_{1}(x) y_{1}-f_{2}(x) y_{2}$,

$\frac{d y_{1}}{d t}=e_{1} f_{1}(x) y_{1}-d_{1} y_{1}$,

$\frac{d y_{2}}{d t}=e_{2} f_{2}(x) y_{2}-d_{2} y_{2}$.

Here, $x, y_{1}$ and $y_{2}$ are the densities of the prey and the two consumers respectively (in milligrams of $\mathrm{C}$ per liter, $(\mathrm{mg} \mathrm{C}) / 1), r$ is the intrinsic growth rate of the prey $\left(\right.$ day $\left.^{-1}\right), d_{1}$ and $d_{2}$ are the specific loss rates of the consumers that include respiration and death $\left(\right.$ day $\left.^{-1}\right)$.

$f_{1}(x)$ and $f_{2}(x)$ are the consumers' ingestion rates, which we assume to follow Holling type II functional response. In other words, $f_{i}(x)$ is a bounded smooth function that satisfies the following assumptions:

$f_{i}(0)=0, \quad f_{i}^{\prime}(x)>0 \quad$ and

$f_{i}^{\prime \prime}(x)<0$ for $x \geqslant 0, \quad i=1,2$.

$e_{1}$ and $e_{2}$ are constant growth efficiencies (conversion rates or yield constants) of converting ingested prey biomass into consumer biomasses. The second law of thermodynamics requires that $e_{1}$ and $e_{2}$ be $<1$. (Note that this assumption of constant growth efficiency together with (2) assures that higher prey density never hurts predator growth.)

$K$ represents a constant carrying capacity that we relate to light in the following way: suppose that we fix light intensity at a certain value, then let the prey (which is a photoautotroph) grow with no consumers but with ample nutrients. The prey density will increase until selfshading ultimately stabilizes it at some value, $K$. Thus, every $K$ value corresponds to a specific limiting light intensity and we might model the influence of higher light intensity as having the effect of raising $K$, all else being equal (Loladze et al., 2000). Much more mechanistic and realistic modeling of light limited growth exists (Huisman and Weissing, 1994; Diehl, 2002). It is possible, however, to show that under suitable parameter choices such more mechanistic models will closely match the dynamics of our simpler model (J. Huisman, pers. commun., derivations available upon request). Because an inclusion of the mechanistic light limited growth would significantly complicate our model, we do not attempt it here.

For simplicity, we will model only two chemical elements, $\mathrm{P}$ and $\mathrm{C}$. The choice of $\mathrm{C}$ is clear, because this element comprises the bulk of the dry weight of the most organisms. Instead of $\mathrm{P}$, however, one can choose any other element as long as it is essential to all species in the system (e.g. N, S, or Ca). (For numerical analysis, however, we will use a particular feature of $\mathrm{P}$ manifested in "the growth rate hypothesis" (Elser et al., 2000), which links species growth rate to species P:C via rRNA machinery.) The cellular physiology of autotrophs allows them to exhibit highly variable elemental composition, mainly because of the presence of a large central vacuole, which is unique to autotroph cells (Sterner and Elser, 2002). For example, iron or calcium concentrations can vary 100 -fold or more in grass forage (Adams, 1974). With regards to $\mathrm{P}$, the green alga Scenedesmus acutus can have cellular P:C ratios (by mass) that range from $1.6 \times 10^{-3}$ to $13 \times 10^{-3}$, almost an order of magnitude (e.g. Elser and Urabe, 1999). On the other hand, in animals $\mathrm{P}: \mathrm{C}$ varies much less within a species (Sterner and Elser, 2002). For example, the zooplankton Daphnia's P:C stays within relatively narrow bounds around $31 \times 10^{-3}$ by mass, decreasing only by around $30 \%$ when food P:C is more than 20 times lower than Daphnia's P:C (Andersen and Hessen, 1991; Sterner and Hessen, 1994; DeMott, 1998). Using only two elements is a gross simplification of reality, but it is a qualitative step forward from conventional "chemically homogeneous" models like model (1). Most importantly, the inclusion of just two elements allows us to explicitly model prey quality by applying the mass balance law.

We express the above considerations in the following assumptions:

(A1): Prey's P:C varies, but never falls below a minimum $q(\mathrm{mg} \mathrm{P} / \mathrm{mg} \mathrm{C})$; the two consumers maintain a constant P:C, $s_{1}$ and $s_{2}(\mathrm{mg} \mathrm{P} / \mathrm{mg} \mathrm{C})$, respectively.

(A2): The system is closed for $\mathrm{P}$, with a total of $P_{T}$ ((mg P)/l), which is divided into two pools: $\mathrm{P}$ in the consumers and the rest as $\mathrm{P}$ potentially available for the prey.

From these two assumptions it follows that $\mathrm{P}$ available for the prey at any given time is $\left(P_{T}-s_{1} y_{1}-\right.$ $\left.s_{2} y_{2}\right)(\mathrm{mg} \mathrm{P}) / 1$. Recalling that $\mathrm{P}: \mathrm{C}$ in the prey should be at least $q(\mathrm{mg} \mathrm{P} / \mathrm{mg} \mathrm{C})$, one obtains that the prey density cannot exceed $\left(P_{T}-s_{1} y_{1}-s_{2} y_{2}\right) / q(\mathrm{mg} \mathrm{C}) / 1$. In the 
spirit of Liebig's Law of the Minimum, the combination of light and $\mathrm{P}$ limits the carrying capacity of the prey to

$\min \left(K, \frac{P_{T}-s_{1} y_{1}-s_{2} y_{2}}{q}\right)$.

To determine prey's P:C at any given time, we follow Andersen (1997) by assuming that the prey can absorb all potentially available $\mathrm{P}$. This assumption is not suitable for modeling multiple competing autotrophs, because uptake of free $\mathrm{P}$ is crucial for the competition outcome and, thus, must be modeled explicitly. For terrestrial systems, it may not be suitable for modeling even one autotroph, because soil is a major stock of P. However, it is not far from reality in freshwater systems, where algae can absorb almost all available $\mathrm{P}$, bringing free phosphate levels in water below detection. And since we neither model multiple preys nor terrestrial systems, we make this assumption, which means that prey's $\mathrm{P}: \mathrm{C}(\mathrm{mg} \mathrm{P}: \mathrm{mg} \mathrm{C})$ at any time is

$\left(P_{T}-s_{1} y_{1}-s_{2} y_{2}\right) / x$.

One issue needs a clarification: since we do not impose the maximal $\mathrm{P}$ quota on the prey, its $\mathrm{P}: \mathrm{C}$ can become potentially unbounded for small $x$ according to (4). As we will show later, this property does not have undesirable effects on the dynamics, because when prey's P:C exceeds that of the consumers (i.e. $>\max \left\{s_{1}, s_{2}\right\}$ ), the consumers become limited by $\mathrm{C}$ and prey's P:C, however large it is, become dynamically irrelevant.

While prey stoichiometry varies according to (4), the $i$ th consumer maintains its constant (homeostatic) P:C, $s_{i}$. If prey's $\mathrm{P}: \mathrm{C}>s_{i}$, then the $i$ th consumer converts consumed prey with the maximal (in $\mathrm{C}$ terms) efficiency $e_{i}$ and egests or excretes any excess of ingested $\mathrm{P}$. If prey's $\mathrm{P}: \mathrm{C}$ is $<s_{i}$, then the $i$ th consumer wastes the excess of ingested $\mathrm{C}$. This waste is assumed to be proportional to the ratio of prey's P:C to $i$ th consumer's P:C (Andersen, 1997), which reduces the growth efficiency in $\mathrm{C}$ terms. The following minimum function provides the simplest way to capture such effects of variable prey quality on consumers' growth efficiency:

$e_{i} \min \left(1, \frac{\left(P_{T}-s_{1} y_{1}-s_{2} y_{2}\right) / x}{s_{i}}\right)$.

An alternative formulation that does not use minimum functions is possible using the concept of synthesizing units (Muller et al., 2001).

The following model simultaneously captures expressions (3) and (5):

$$
\begin{aligned}
\frac{d x}{d t}= & r x\left(1-\frac{x}{\min \left(K,\left(P_{T}-s_{1} y_{1}-s_{2} y_{2}\right) / q\right)}\right) \\
& -f_{1}(x) y_{1}-f_{2}(x) y_{2},
\end{aligned}
$$

$$
\begin{aligned}
\frac{d y_{1}}{d t}= & e_{1} \min \left(1, \frac{\left(P_{T}-s_{1} y_{1}-s_{2} y_{2}\right) / x}{s_{1}}\right) \\
& \times f_{1}(x) y_{1}-d_{1} y_{1}, \\
\frac{d y_{2}}{d t}= & e_{2} \min \left(1, \frac{\left(P_{T}-s_{1} y_{1}-s_{2} y_{2}\right) / x}{s_{2}}\right) \\
& \times f_{2}(x) y_{2}-d_{2} y_{2} .
\end{aligned}
$$

Note, that the prey's growth rate in the absence of consumers,

$\frac{d x}{d t}=r x\left(1-\frac{x}{\min \left(K,\left(P_{T}-s_{1} y_{1}-s_{2} y_{2}\right) / q\right)}\right)$,

can be equivalently represented as the minimum of a logistic equation and of a biomass version of Droop's (1974) model:

$$
\begin{aligned}
\frac{d x}{d t}= & \min \left(r x\left(1-\frac{x}{K}\right),\right. \\
& \left.r x\left(1-\frac{q}{\left(P_{T}-s_{1} y_{1}-s_{2} y_{2}\right) / x}\right)\right) .
\end{aligned}
$$

\section{Analysis}

\subsection{Analytical analysis}

It is easy to see why conventional model (1) almost never has a positive equilibrium, i.e. an equilibrium with all three species coexisting. If such an equilibrium exists, then the prey density, $x^{*}$, at such an equilibrium should simultaneously satisfy the following two equations to keep consumers' net growth rates equal to zero:

$f_{1}\left(x^{*}\right)=\frac{d_{1}}{e_{1}}$ and $f_{2}\left(x^{*}\right)=\frac{d_{2}}{e_{2}}$,

which is almost impossible - the set of parameter values satisfying both equations is of measure zero. Moreover, Armstrong and McGehee (1980) rigorously showed that for the class of models, to which model (1) belongs, an attracting positive equilibrium is not only almost impossible, but is absolutely impossible with $n$ species on fewer than $n$ resources. Nevertheless, we will show that for our stoichiometrically explicit model (6), which does not belong to any of the classes analyzed by Armstrong and McGehee (1980), such an equilibrium exists.

Appendix A.1 shows that the model (6) is well defined (this includes the case when $x \rightarrow 0$ ) and that solutions to initial values are unique.

Stoichiometric considerations provide natural bounds on densities of all species. Phosphorus sets a clear upper bound: the sum of $\mathrm{P}$ in the prey and the two consumers cannot exceed $P_{T}$-the total $\mathrm{P}$ in the system. This property, together with the invariance of solutions in forward time, is proved in the following theorem. 
Theorem 1. Let $k=\min \left(K, P_{T} / q\right)$. Then, solutions with initial conditions in

$$
\begin{aligned}
\Delta \equiv & \left\{\left(x, y_{1}, y_{2}\right): 0<x<k, 0<y_{1}, 0<y_{2}, q x+s_{1} y\right. \\
& \left.+s_{2} y<P_{T}\right\}
\end{aligned}
$$

remain there for all forward times.

Proof is in Appendix A.2.

By $\breve{\Delta}$, we denote the region $\Delta$ with its entire boundary except of the biologically unrealistic edge $\gamma=$ $\left\{\left(x, y_{1}, y_{2}\right): x=0, \quad P_{T}=s_{1} y_{1}+s_{2} y_{2}\right\}$. (At this edge consumers contain all $\mathrm{P}$, thus there is no prey in the system.) That is,

$\breve{\Delta}=(\partial \Delta \backslash \gamma) \cup \Delta$.

To simplify our analysis, we rewrite system (6) in the following form:

$x^{\prime}=x F\left(x, y_{1}, y_{2}\right)$,

$y_{1}^{\prime}=y_{1} G_{1}\left(x, y_{1}, y_{2}\right)$,

$y_{2}^{\prime}=y_{2} G_{2}\left(x, y_{1}, y_{2}\right)$,

where

$$
\begin{aligned}
F\left(x, y_{1}, y_{2}\right)= & r\left(1-\frac{x}{\min \left(K,\left(P_{T}-s_{1} y_{1}-s_{2} y_{2}\right) / q\right)}\right) \\
& -\sum_{i=1}^{2} \frac{f_{i}(x)}{x} y_{i},
\end{aligned}
$$

$$
\begin{aligned}
G_{i}\left(x, y_{1}, y_{2}\right) \\
=e_{i} \min \left(1, \frac{\left(P_{T}-s_{1} y_{1}-s_{2} y_{2}\right) / x}{s_{i}}\right) f_{i}(x)-d_{i} \\
=e_{i} \min \left(f_{i}(x), \frac{P_{T}-s_{1} y_{1}-s_{2} y_{2}}{s_{i}} \frac{f_{i}(x)}{x}\right) \\
\quad-d_{i}, \quad i=1,2
\end{aligned}
$$

are per capita net growth rates of the prey and the two consumers, respectively.

Condition (A.1) ensures that all partial derivatives of $F$ and $G_{i}$ exist almost everywhere on $\breve{\Delta}$ :

$$
\begin{aligned}
\frac{\partial F}{\partial x}= & -\frac{r}{\min \left(K,\left(P_{T}-s_{1} y_{1}-s_{2} y_{2}\right) / q\right)} \\
& -\sum_{i=1}^{2}\left(\frac{f_{i}(x)}{x}\right)^{\prime} y_{i} .
\end{aligned}
$$

For $i, j=1,2$, we have

$$
\frac{\partial F}{\partial y_{i}}= \begin{cases}-\frac{f_{i}(x)}{x}<0 & \text { if } K<\frac{P_{T}-s_{1} y_{1}-s_{2} y_{2}}{q}, \\ -\frac{r q s_{i} x}{\left(P_{T}-s_{1} y_{1}-s_{2} y_{2}\right)^{2}} & \text { if } K>\frac{P_{T}-s_{1} y_{1}-s_{2} y_{2}}{q}, \\ -\frac{f_{i}(x)}{x}<0 & \end{cases}
$$

$$
\frac{\partial G_{i}}{\partial x}=\left\{\begin{array}{cc}
e_{i} f_{i}^{\prime}(x)>0 & \text { if } \frac{P_{T}-s_{1} y_{1}-s_{2} y_{2}}{x}>s_{i}, \\
e_{i} \frac{P_{T}-s_{1} y_{1}-s_{2} y_{2}}{s_{i}} & \\
\times\left(\frac{f_{i}(x)}{x}\right)^{\prime}<0 & \text { if } \frac{P_{T}-s_{1} y_{1}-s_{2} y_{2}}{x}<s_{i},
\end{array}\right.
$$

$$
\frac{\partial G_{i}}{\partial y_{j}}= \begin{cases}0 & \text { if } \frac{P_{T}-s_{1} y_{1}-s_{2} y_{2}}{x}>s_{i} \\ -e_{i} \frac{s_{j}}{s_{i}} \frac{f_{i}(x)}{x}<0 & \text { if } \frac{P_{T}-s_{1} y_{1}-s_{2} y_{2}}{x}<s_{i}\end{cases}
$$

An unusual and very important property of this system is that the sign of $\partial G_{i} / \partial x, i=1,2$ changes from positive to negative as soon as prey's P:C (4) is < P:C of the $i$ th consumer, $s_{i}$, as (14) shows. These negative derivatives mean that, all else being equal, higher prey density reduces growth rates of consumers! This effect is in sharp contrast to many population dynamics models, which routinely assume that higher prey density cannot hurt predator growth. Note that neither we imposed this effect on the model nor did we explicitly built it in any of our assumptions; it follows from the stoichiometric properties of model (6).

An intuitive explanation for this effect is following: if prey quality is bad, i.e. if

$\left(P_{T}-s_{1} y_{1}-s_{2} y_{2}\right) / x<s_{i}, \quad i=1,2$

then any increase in the prey density, $x$, further deteriorates prey quality, which offsets any benefit that higher prey density may provide to already $\mathrm{P}$ limited consumers. Let us take a more rigorous look at the effect of prey quality on the species interactions and coexistence.

If prey quality is good for both consumers, i.e. if

$\left(P_{T}-s_{1} y_{1}-s_{2} y_{2}\right) / x>s_{i}, \quad i=1,2$

then equations for consumer growth ((6b), (6c)) degenerate to those in conventional system $((1 \mathrm{~b}),(1 \mathrm{c}))$ and, according to conditions (8), an equilibrium is almost impossible. Moreover, as Appendix A.3 shows, at such an unlikely equilibrium the ecosystem matrix (it is not a Jacobian but matrix (A.9), where the $i j$ th term reflects the effect of the $j$ th species on the $i$ th species per capita growth rate) takes this form:

$$
\left(\begin{array}{ccc}
+/- & - & - \\
+ & 0 & 0 \\
+ & 0 & 0
\end{array}\right)
$$

meaning that conventional predator-prey, i.e. $(+,-)$ type, interactions exist between the consumers and the prey.

The picture radically changes if prey quality is bad, i.e. if (16) hold. Stable coexistence becomes a viable possibility: Appendix A.3 contains analytical 
Table 1

Parameters (P) of model (6) with their values (V) used for numerical simulations.

\begin{tabular}{|c|c|c|c|}
\hline P & Description & $\mathrm{V}$ & Units \\
\hline$r$ & Intrinsic growth rate of the prey & 0.93 & $\mathrm{day}^{-1}$ \\
\hline$K$ & Resource carrying capacity determined by light & $0-1.75$ & $(\mathrm{mg} \mathrm{C}) / 1$ \\
\hline$c_{1}$ & Maximal ingestion rate of the 1 st consumer & 0.7 & $\operatorname{day}^{-1}$ \\
\hline$c_{2}$ & Maximal ingestion rate of the 2 nd consumer & 0.8 & day $^{-1}$ \\
\hline$a_{1}$ & Half-saturation constant of the 1 st consumer & 0.3 & $(\mathrm{mg} \mathrm{C}) / 1$ \\
\hline$a_{2}$ & Half-saturation constant of the 2 nd consumer & 0.2 & $(\mathrm{mg} \mathrm{C}) / 1$ \\
\hline$e_{1}$ & Maximal conversion rate of the 1 st consumer & 0.72 & \\
\hline$e_{2}$ & Maximal conversion rate of the 2 nd consumer & 0.76 & \\
\hline$d_{1}$ & Loss rate of the 1 st consumer & 0.23 & $\operatorname{day}^{-1}$ \\
\hline$d_{2}$ & Loss rate of the 2 nd consumer & 0.2 & $\operatorname{day}^{-1}$ \\
\hline$s_{1}$ & Constant P:C of the 1st consumer & 0.032 & $(\mathrm{mg} \mathrm{P}) /(\mathrm{mg} \mathrm{C})$ \\
\hline$s_{2}$ & Constant P:C of the 2 st consumer & 0.05 & $(\mathrm{mg} \mathrm{P}) /(\mathrm{mg} \mathrm{C})$ \\
\hline$q$ & Minimal possible P:C of the prey & 0.004 & $(\mathrm{mg} \mathrm{P}) /(\mathrm{mg} \mathrm{C})$ \\
\hline$P_{T}$ & Total $\mathrm{P}$ in the system & 0.03 & $(\mathrm{mg} \mathrm{P}) / 1$ \\
\hline
\end{tabular}

expressions for equilibrium values ((A.10), (A.11)), and the next sections shows numerically that such an equilibrium is a structurally stable attracting node. Eq. (A.10) and (A.11) suggest an interesting dependence of equilibrium values on light intensity: increasing energy flow into this food web (i.e. increasing parameter $K$ ), does not change prey density, but can increase density of one consumer and hurt the other one. In the next section, a bifurcation diagram shows exactly such a pattern.

Moreover, under bad prey quality conditions, ecosystem matrix takes the following form (Appendix A.3 contains the derivations and also covers boundary equilibria):

$$
\left(\begin{array}{ccc}
+/- & - & - \\
- & - & - \\
- & - & -
\end{array}\right)
$$

meaning that all three species compete with each other, i.e. interactions between them are $(-,-)$ type. Note also that each consumer interferes with itself and the other consumer. It has been shown that interference among consumers can enhance possibilities for coexistence (Vance, 1985; Kuang et al., 2003).

Finally, prey quality can be bad for one consumer, but good for the other. In this case, one consumer will be in predator-prey relationship with the prey, while the other will be competing with the prey. The next section presents a stable equilibrium for such a case as well. The above analysis illustrates that changes in prey stoichiometry can fundamentally alter interactions in this simple food web.

\subsection{Numerical analysis}

To numerically illustrate the stable coexistence of all three species and to further the analysis of model (6), we choose both $f_{i}(x), i=1,2$ as Monod (MichaelisMenten) functions:

$f_{i}(x)=\frac{c_{i} x}{a_{i}+x}, \quad i=1,2$.

Table 1 lists parameter values within biologically plausible range (Urabe and Sterner, 1996; Andersen, 1997; Elser and Urabe, 1999). These values indicate that the second consumer needs more $\mathrm{P}\left(s_{2}>s_{1}\right)$ but, as a trade-off, has a higher growth rate $\left(e_{2} f_{2}(x)>e_{1} f_{1}(x)\right.$ for $x>0)$ if prey quality is good. Such a trade-off reflects "the growth rate hypothesis" (Elser et al., 2000) and has been empirically supported in studies of zooplankton (Main et al., 1997). Our goal here is only to illustrate that coexistent equilibrium is possible and that it can be asymptotically stable (with a large attraction basin) as well as structurally stable. Although such an equilibrium can arise for parameters with biologically meaningful values, we leave open the question of how widespread such type of coexistence is in nature. We would very much want to relate our numerical simulations to actual laboratory or field experiments on the effects of variable prey stoichiometry on competition among herbivorous taxa, but we are not aware of any such experiments.

The most interesting issue seems to be the dependence of coexistence on light intensity, i.e. energy flow into the system. Light intensity can change prey quality (e.g. Urabe and Sterner, 1996), and as analytical analysis in the previous section suggests, such change in prey's P:C can profoundly affect this food web and the competition between consumers. Bifurcation diagrams provide a clear and visual way to understand how the behavior of a dynamical system depends on a parameter. In Fig. 1, we present such a diagram, where species densities are drawn along the gradient of light intensity implicitly represented by $K$.

When the light intensity is low to medium (hereafter values are rounded to the nearest hundredth), 


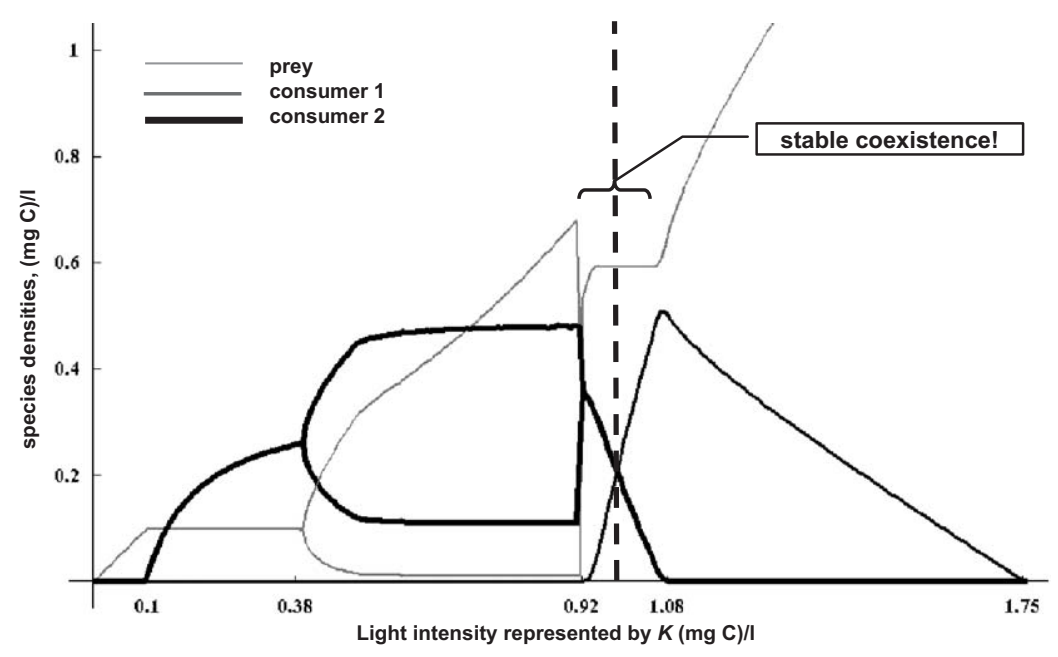

Fig. 1. Both consumers can stably coexist on a single prey, while prey quality (prey's P:C) limits them both $(0.92<K<1.08)$. This is a bifurcation diagram of all three species densities plotted (after transients) against parameter $K$, where $K$ indirectly reflects energy (light) input into the system. Each line represents species density at a stable equilibrium, except when it splits into two lines to represent the maximum and the minimum species densities at a stable limit cycle. All parameters are as in Table 1, but $K$ varies from 0 to 1.8 (all initial densities that we tried lead to the picture). For $K<0.09$, neither consumer persists due to very low food quantity, but for $K>1.75$ - due to very low food quality. As $K$ grows from 0.09 to 0.38 , a consumer with higher intrinsic growth rate (and higher P:C) increases its density. But at $K=0.38$, the stable equilibrium with this consumer and the prey loses its stability to a limit cycle, an amplitude of which grows with $K$ (from 0.38 to 0.92 ). The system here exhibits "the paradox of enrichment". This trend stops at $K=0.92$, when the limit cycle disappears and the consumer with a lower P:C (and lower intrinsic growth rate) invades. All three species coexist at this newly emerged stable equilibrium. However, as $K$ further grows from 0.92 to 1.08 , worsening food quality gradually lowers the density of P-rich consumer and, despite abundant food quantity, eventually drives this consumer to a deterministic extinction ("the paradox of energy enrichment"). As $K$ increases from 1.08 to 1.75 , the other consumer meets the same fate. A vertical dotted line represents an equilibrium at $K=1$, which also is shown in Fig. 2.

$0<K<0.92$, the prey quality is sufficiently high and the dynamics of the system matches closely the behavior of conventional model (1) with $f_{i}(x)$ given by $(20)$. In particular, for $0<K<0.09$, none of the consumers can survive due to low food abundance. For $0.09<K<0.92$, the CEP holds and the consumer with lower break-even prey concentration wins. Note that at $K=0.38$, the system undergoes Rosenzweig's (1971) paradox of enrichment via Hopf bifurcation: stable equilibrium loses its stability to a limit cycle, the period of which increases with $K$. This period would have continued to increase with $K$ for a conventional system (1), but a paradoxical situation happens in system (6): under high light intensity, $0.92<K<1.08$, both consumers coexist at a stable equilibrium exploiting a single prey! This is possible because high light intensity lowers prey quality to the point, where the consumer with lower $\mathrm{P}$ demand is able to invade the system. Note that as $K$ increases from 0.92 to 1.08 , the prey equilibrium density does not change, but the density of P-rich consumer declines, while that of P-poor consumer increases. Our analytical analysis suggested exactly such a pattern. In the next section, we show that at this equilibrium both consumers are limited by the $\mathrm{P}$ content of the prey.

All three species coexist at an equilibrium for any $K \in(0.92,1.08)$. Let us consider closer such an equilibrium, for one value of $K$ in this interval. For example, for $K=1$, the system has the following positive equilibrium:

$$
\left(x, y_{1}, y_{2}\right)=(0.59,0.26,0.17) \text {. }
$$

A dotted line in Fig. 1 indicates this equilibrium. Fig. 2 shows how the system approaches this equilibrium over time. Calculations show that all eigenvalues are real and negative meaning that the equilibrium is a locally asymptotically stable node. From (A.10), (A.11), and (20), it follows that this equilibrium is unique. It can be shown that all boundary equilibria are unstable and the system is persistent. (That is, all species, if present, will coexist. This also implies that the invasions of either consumer species will be successful.) Moreover, all our numerical simulations indicate that trajectories with positive initial conditions in $\Delta$ converge to equilibrium (21). Numerical analysis also shows that varying every other parameter around the value given in Table 1, does not destroy the coexistence at a stable equilibrium: for example, for $P_{T}$ such an interval is $(0.026,0.032)$, and for $q$ it is $(0,0.012)$. In other words, this stable node is also structurally stable.

A peculiar intermediate scenario can occur, when prey quality is bad for P-rich consumer (i.e. a consumer with a higher P:C ratio, $s_{i}$ ), but good for P-poor consumer. Fig. 3 shows a bifurcation diagram for the indicated therein parameter set, where all species coexist at fixed densities for any $K \in(0.35,0.7)$. For this interval of light intensity, prey's P:C (4) lies between P:C of the two 


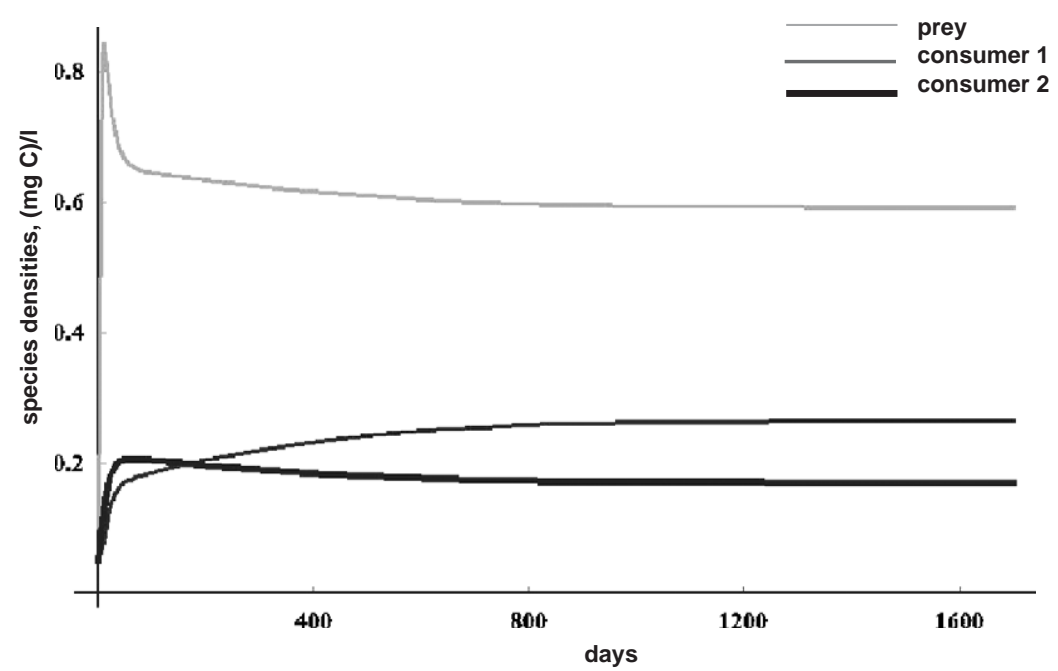

Fig. 2. The densities of each species plotted against time. Parameters are in a biologically realistic range as in Table 1 and $K=1$ (the initial conditions are $\left.x=y_{1}=y_{2}=0.1(\mathrm{mg} \mathrm{C}) / \mathrm{l}\right)$. Both consumers are limited by the resource quality and the species densities eventually settle to fixed values. A vertical dotted line indicates this equilibrium in Fig. 1.

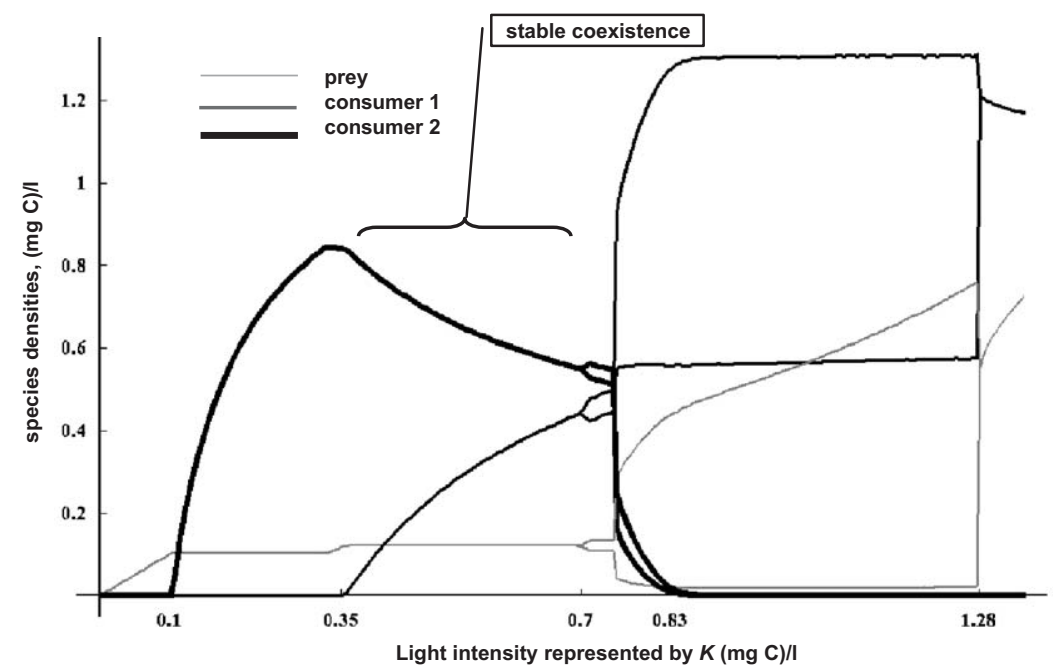

Fig. 3. Both consumers can stably coexist at an equilibrium, but unlike Fig. 1, prey quantity limits P-poor consumer and prey quality limits P-rich one. An explanation of the diagram as in Fig. 1, but for the following parameters: $r=1.4, c_{1}=0.6, c_{2}=0.63, a_{1}=0.36, a_{2}=0.45, e_{1}=0.8$, $e_{2}=0.85, P_{T}=0.036, q=0.003, s_{1}=0.025, s_{2}=0.038, d_{1}=0.12, d_{2}=0.1$ and $K$ ranging from 0 to 1.4 . Not only all three species can coexist at a stable equilibrium $(0.35<K<0.7)$, but also they can coexist via oscillations $(0.7<K<0.83)$. For $K<0.1$, neither consumer can persist due to very low food quantity, but for $K>0.83$, P-rich consumer cannot persist due to very low food quality (very low prey's P:C).

consumers, $s_{1}$ and $s_{2}$. Interestingly, for higher light intensity, $K \in(0.7,0.83)$, both consumers still coexist, but in an oscillatory fashion with prey quality limiting P-poor consumer as well.

\subsection{Graphical analysis}

Since system (6) involves three differential equations, its phase space is three dimensional. First, let us consider a simpler two-dimensional case: a single consumer on one prey. This case was rigorously analyzed in Loladze et al. (2000) using the method of nullclines. A nullcline (or Zero Net Growth Isoclines (ZNGI), Tilman, 1982) is the set of points (on the phase plane) at which species' growth rate is zero. Hence, if nullclines of all species intersect at some common point, then the growth rate of every species is zero at such a point, making it an equilibrium. The stability type of such an equilibrium can sometimes be determined by the way nullclines intersect (e.g. Rosenzweig, 1971; Loladze et al., 2000). As we see in Fig. 4, the resource nullcline is hump shaped, a usual feature of many predator-prey models. The consumer nullcline, however, has an unusual shape of a right triangle (in conventional consumer-resource 


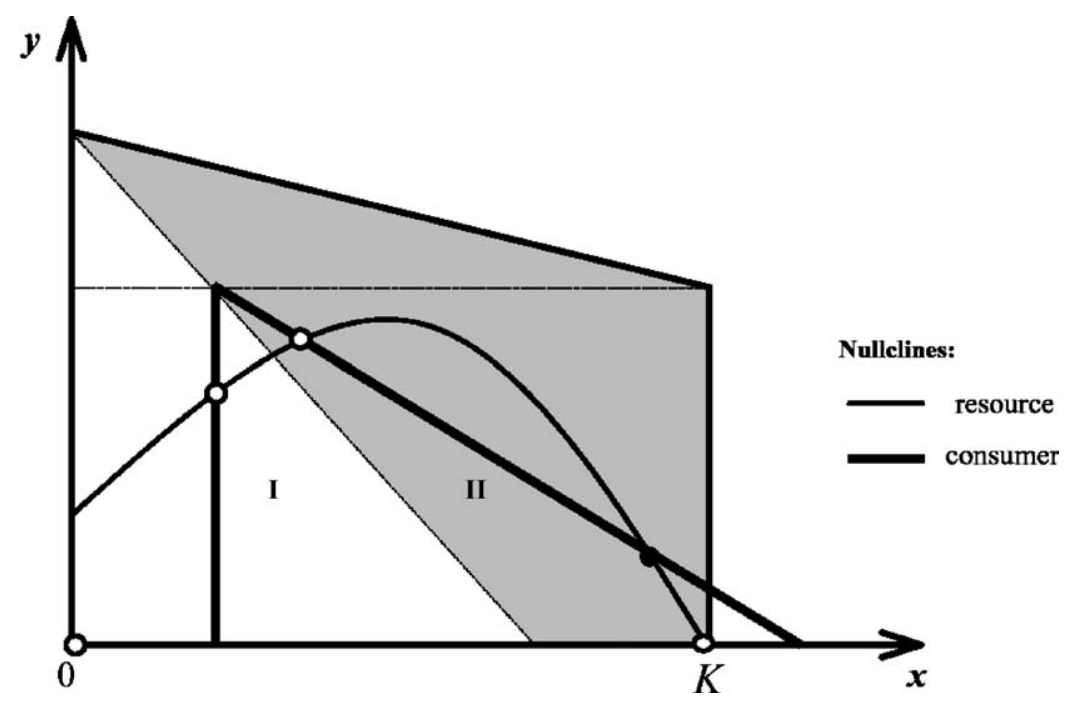

Fig. 4. (from Loladze et al. (2000)). Stoichiometric properties confine dynamics of one consumer-one prey model to a trapezoid-shaped area and divide the phase plane into two regions. In region I, like in classical Lotka-Volterra model, food quantity limits consumer growth. In shaded Region II, food quality (prey's P:C) constrains consumer growth. Competition for P between the consumer and the prey alters their interactions from $(+,-)$ in region I to $(-,-)$ in region II. This bends the consumer nullcline in region II. This shape of the consumer nullcline, with two $x$-intercepts, creates the potential for multiple positive steady states. A solid circle denotes a stable equilibrium, clear circles - unstable equilibriums.

models, it is a vertical line or an increasing curve). Here, multiple equilibria are possible because two nullclines with such shapes can intersect more than once. The vertical segment of the consumer nullcline lies in a region where the prey quantity limits the consumer like in conventional predator-prey models. The slanted segment of the consumer nullcline lies in a region where prey quality limits the consumer and novel dynamics arise.

Introducing the second consumer adds a third dimension to Fig. 4 and, instead of nullclines on the phase plane, we have nullsurfaces in the phase space. But we can continue analysis on the plane by viewing a slice of the phase space passing through an equilibrium at which all three species stably coexist. For example, for equilibrium (21), the slice produced by plane $y_{1}=0.26$ $(\mathrm{mg} \mathrm{C}) / 1$ yields Fig. 5 (slicing with plane $y_{2}=0.17$ yields a similar picture). Fig. 5, similar to Fig. 4, shows that coexisting consumers are limited by prey quality, because equilibrium lies on the slanted part of each consumer's nullsurfaces. Indeed, at equilibrium (21), inequalities (16) hold, implying that both consumers are limited by the same element in the prey. Chesson (2000), discussing stable coexistence, points out that it "necessarily requires important ecological differences between species and that often involve trade-offs". There is such a trade-off in our case. P-rich consumer has a lower break-even concentration, i.e. $R^{*}$ value (Tilman, 1982) or the smallest $x$-intercept (Fig. 5). Consequently, this consumer wins when food quantity limits both consumers. However, as Fig. 5 shows, P-poor consumer, as a trade-off, has larger second $x$-intercept. By analogy with $R^{*}$, we can denote this intercept as $Q^{*}$ ( $Q$ for "quality"), because it determines the lowest food quality $\left(P_{T} / Q^{*}\right)$ that can support a consumer in the absence of competitors. For $i$ th consumer, $R^{*}$ and $Q^{*}$ are the solutions of the following equation:

$e_{i} \min \left(1, \frac{P_{T} / x}{s_{i}}\right) f_{i}(x)-d_{i}=0, \quad i=1,2$.

(Because Fig. 5 is a slice of the phase space, actual $Q^{*}$ values for both consumers are higher than depicted $x$ intercepts, 1.62 for P-rich consumer and 1.75 for P-poor one.) The trade-off between $R^{*}$ and $Q^{*}$ values allows two consumers to stably coexist.

Another way to look at the equilibrium coexistence is to use the perspective of the resource competition theory (Leon and Tumpson, 1975; Tilman, 1982; Grover, 1997), where nullclines, i.e. ZNGIs, are drawn on the plane with each axes representing availability of one abiotic nutrient. For the case of essential nutrients, nullcline for each consumer is $\mathrm{L}$ shaped. Because the vertical segments of these two L's are parallel, they cannot intersect; the same is true for horizontal segments. That is why in the resource competition theory, stable coexistence is impossible for both consumers if both are limited by the same nutrient. Analogously, we can draw such nullclines for consumers in system (6) on a plane with axes representing availability of $\mathrm{C}$ and P. Fig. 6 shows that, instead of being L-shaped, such nullclines take the shape of tilted V's, where the angle of the tilt is species-specific. The segments representing $\mathrm{P}$ limitation on the two $\mathrm{V}$ nullclines intersect to yield a stable equilibrium. This provides graphical explanation of why prey's $\mathrm{P}$ content can limit both consumers at the coexistent equilibrium. 


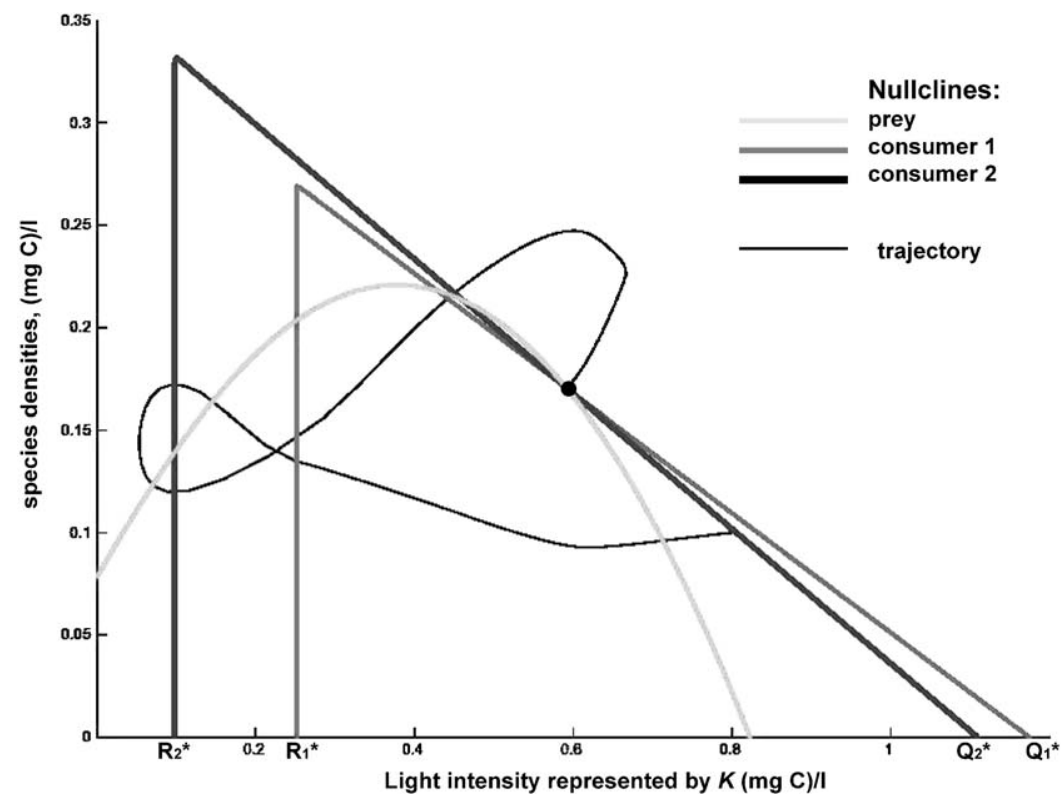

Fig. 5. For $K=1$, slicing the phase space at equilibrium $(0.59,0.26,0.17)$ using plane $y_{1}=0.26$ produces picture analogous to Fig. 4 . The nullsurface of each consumer has two $x$-intercepts: the one closer to the origin is consumer's $R^{*}$ value, which determines the winner when the system is governed by food quantity; the other $x$-intercept is consumer's $Q^{*}$ value, which defines the lowest food quality $\left(P_{T} / Q^{*}\right)$ that can support the consumer in the system with no competitors. The trade-off between $R^{*}$ value (the smaller is better) and $Q^{*}$ value (the larger is better) allows two consumers to stably coexist.

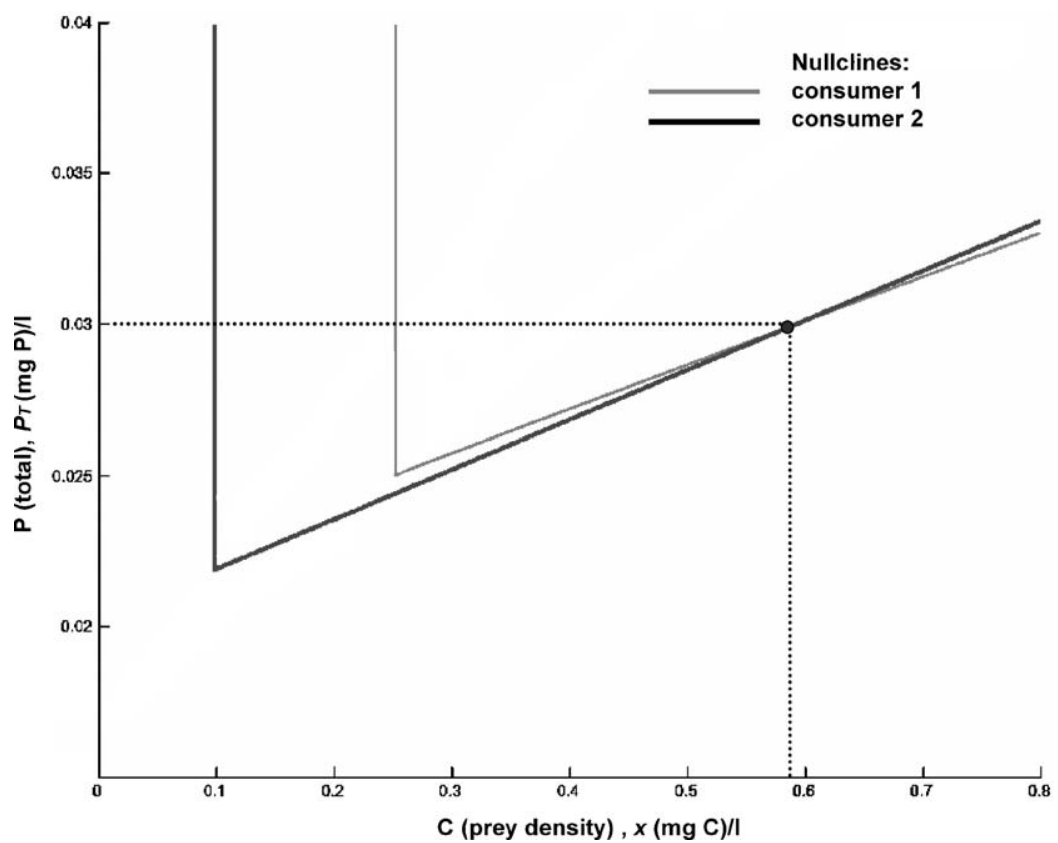

Fig. 6. Analogous to the resource competition theory, we draw consumer nullclines on the $\mathrm{C} \times \mathrm{P}$ phase plane. Instead of usual L shapes, the nullclines take the shape of tilted $\mathrm{V}$, where the angle of the tilt depends on consumer's stoichiometric properties. This shape can lead to an attracting equilibrium as shown, where both consumers coexist, even though they both are limited by the same chemical element in the prey-P.

\section{Discussion}

We showed that chemical heterogeneity within and among species can promote species coexistence, at least in the simple food web analyzed above. In particular, two consumers exploiting a single prey can coexist at a stable equilibrium. Such an equilibrium is also structurally stable, i.e. slight variations in the values of any of the parameters do not destroy it. This result is surprising in the light of the CEP, where a stable equilibrium is impossible in a homogeneous system with $n$ consumers exploiting fewer than $n$ preys. Since the CEP is a 
rigorous theoretical result (Armstrong and McGehee, 1980), one may ask why our result is mathematically possible at all. Our model, unlike conventional resource based models, does not assume a $(+,-)$ relationship between consumers and preys nor does it have linear growth assumptions as in Levin's (1970) "limiting factor" model. Instead, it relies on the fact that all species share common essential chemical elements, but in different proportions. Consumer species differ in their chemical constitution, both from each other and from the resources they consume. Model (6) captures this by considering consumers with distinct $\mathrm{P}: \mathrm{Cs}$, while allowing the $\mathrm{P}: \mathrm{C}$ of autotrophic prey to vary, as it does in nature. It is a fact that any prey contains several chemical elements that are essential to all of its consumers (e.g. C, $\mathrm{N}, \mathrm{P}, \mathrm{S}, \mathrm{K}$, and $\mathrm{Ca}$ ). The concentrations of these elements, however, vary significantly in autotrophs (Sterner and Elser, 2002; Adams, 1974), which makes autotrophs into channels of variable quality through which multiple nutrients flow to their consumers. Altering the stoichiometry of these channels can profoundly affect food webs and species coexistence.

Stoichiometric constraints naturally bound population densities: every organism requires at least some amount of each essential chemical element. Therefore, the total amount of this element in the system limits the overall biomass as shown in Theorem 1 for P. All three species in our system, and in fact, all species in any ecosystem, share a common need for C and P. Such shared requirements for essential elements connect population characteristics to ecosystem properties, and create important feedbacks between these two levels of organizational complexity. Theorem 1 proves one example of such dependence: all dynamics in the system are confined to a region defined by the interplay between ecosystem properties (total $\mathrm{P}$, light intensity) and species-specific stoichiometries (the consumers' P:C and the prey's minimal P:C). Moreover, the prey's carrying capacity (Eq. (3)) is not static, as is assumed in the logistic equation (contained in (1a)), but instead is a dynamic quantity that depends on consumer densities, energy inflow, and total $\mathrm{P}$.

In addition to providing biologically meaningful bounds on the dynamics, the inclusion of multiple elements and their ratios yields novel dynamics within these bounds. When prey quality is good, the sheer quantity of the resource limits consumers. The resource competition theory completely predicts the dynamics: the consumer with the lowest break-even concentration wins (see Eq. (8) and Fig. 1, when $0<K<0.92$ ). However, when prey quality, is bad, a novel attracting equilibrium can arise (determined by Eqs. (A.10) and (A.11) and shown in Fig. 1, when $0.92<K<1.08$ ) with all three species coexisting.

We caution, however, that although stoichiometric considerations enhance possibilities for coexistence at an equilibrium, nevertheless such possibilities are limited. For example, coexistence at a stable equilibrium is impossible when we add a third competitor to system (6) but continue to track only two elements, $\mathrm{C}$ and $\mathrm{P}$. This may suggest that the CEP is still valid, because still at most two consumers can coexist on two chemical elements (abiotic resources) that are just packaged in one prey. Abrams (1988), discussing the problem of counting resources in the context of the CEP, stated that "it would clearly be inappropriate to define resources in such a way that the theory [of the CEP] is simply false". Given our result then, for the CEP to hold, one should not count a prey species as a single resource but as a source of multiple abiotic resources to all its consumers. However, even with such considerations, the analyzed equilibrium (21) is intriguing, because it exists with two consumers simultaneously limited by a single essential element in the prey-P. In addition, at such an equilibrium, the two consumers and the prey are all competitors (as the negative signs in the ecosystem matrix (19) indicate), and one can argue that three competitors coexist on just two distinct abiotic resources, $\mathrm{C}$ and $\mathrm{P}$.

Such coexistence is achieved in model (6) without any spatial heterogeneity and with no external fluctuations. Instead, chemical heterogeneity within and among species alone provides new mechanisms for coexistence. This raises a question of the role of such heterogeneity in supporting the puzzling biodiversity on Earth. Note that the bad quality of the prey weakens consumer-prey interactions by limiting $\mathrm{C}$ (energy) flow from the prey into the consumer biomass. As we have shown here (Figs. 1 and 3), such weakening promotes coexistence, a result that agrees with "the weak interaction strength" hypothesis (McCann et al., 1998). This suggests that ecological stoichiometry may play a role in the biodiversitystability debate (McCann, 2000), particularly if multiple chemical elements are considered. Though $\mathrm{P}$ is of special interest because it appears tightly linked to growth rates of individual organisms via P-rich rRNA, which is required in high concentration to achieve rapid growth rate (Elser et al., 1996), other elements (e.g. N, Ca, Fe, I) may also have important effects on ecological interactions (Sterner and Elser, 2002; Milewski and Diamond, 2000; Williams and Frausto da Silva, 2001). For example, Daufresne and Loreau (2001a) investigated how the difference in N:P ratio between plants and herbivores affects plant nutrient limitation.

There are other areas of population dynamics and ecology where ecological stoichiometry may provide new insights. Chemostat theory is one of the most thoroughly studied parts of population dynamics (e.g. Hsu et al., 1981; Smith and Waltman, 1995; Li et al., 2000). This theory, in which nutrients are central, 
is particularly suitable for stoichiometric extensions. Another interesting avenue is a coupling of stoichiometric effects with spatial ones. Work by Fagan and Bishop (2000) on Mount St. Helens suggests that nutritional quality of invading plants may depend on their spatial position, leading to differential effects on herbivores and on the dynamics of primary succession. Codeco and Grover (2001) provided another starting point by considering the effects of various P:C supply ratios on competition between algae and bacteria in a gradostat (interconnected multiple chemostats). Fryxell (1991) investigated how forage quality may affect spatial distribution of herbivores. Fryxell did not express forage quality as stoichiometric ratios but instead used digestibility as a plant quality indicator. Other possible indicators could be plant toxicity or resistance to grazing, and all these indicators are not necessarily mutually exclusive. Daufresne and Loreau (2001b) provided the first application of stoichiometric principles for modeling of autotroph-decomposer communities. Grover (2002) investigated how stoichiometric constraints, coupled with grazing pressure, affect coexistence of two prey species on two resources. Work by Diehl (2003) is an original application of stoichiometric models to the evolution of omnivory. By incorporating resource stoichiometry into our understanding of consumer-resource dynamics, it may eventually be possible to characterize opportunities for consumer coexistence in terms of temporal variability in resource quality much the way spatial heterogeneity has proven critical to coexistence in some systems.

Understanding how the changing balance of chemical elements affects populations and ecosystems becomes ever more important as human activities profoundly alter the global cycles of $\mathrm{C}, \mathrm{P}$ and other elements essential for all life such as $\mathrm{N}$ and $\mathrm{S}$. These changes can alter the stoichiometry of plants and algae-the foundation of virtually all food webs in nature. For example, it is well established that elevated carbon dioxide $\left(\mathrm{CO}_{2}\right)$, on average, lowers $\mathrm{N}: \mathrm{C}$ ratio in plants (Cotrufo et al., 1998; Jablonski et al., 2002). It has recently been shown that elevated $\mathrm{CO}_{2}$, similarly to increased light intensity, can decrease $\mathrm{P}: \mathrm{C}$ ratio in algae (Urabe et al., 2003) and can also alter concentrations of other vital macro- and micro-elements in wild and cultivated plants (Loladze, 2002). How might such changes affect species interactions and Earth's biodiversity? In model (6) with only two chemical elements, increase in light intensity changed the stoichiometry of one prey species, which in turn profoundly affected the coexistence of two consumers. It is plausible that similar effects take place in real ecosystems consisting of multiple elements and multitude of species, in which anthropogenic impacts are likely altering the nutrient content of autotrophs at the base of Earth's food webs.

\section{Acknowledgments}

We thank Chris Klausmeier and two anonymous reviewers for comments. This research has been partially supported by NSF Grant DMS-0077790 and IBN9977047. I.L. also has been partially supported by NSF Grant DEB-0083566 and Andrew W. Mellon Foundation.

\section{Appendix A}

\section{A.1. System (6) is well defined}

Proof. From conditions (2) it follows that for $f_{i}(x) / x$ the following holds:

$$
\begin{aligned}
& \lim _{x \rightarrow 0} \frac{f_{i}(x)}{x}=f^{\prime}(0)<\infty \quad \text { and } \\
& \left(\frac{f_{i}(x)}{x}\right)^{\prime}<0 \text { for } x>0, \quad i=1,2 .
\end{aligned}
$$

Thus, $y_{i}^{\prime}(t)$ is well defined as $x \rightarrow 0$, since for $i=1,2$,

$$
\begin{aligned}
& \min \left(1, \frac{P_{T}-s_{1} y_{1}-s_{2} y_{2}}{x}\right) f_{i}(x) \\
& =\min \left(f_{i}(x), \frac{\left(P_{T}-s_{1} y_{1}-s_{2} y_{2}\right) f_{i}(x)}{x}\right) .
\end{aligned}
$$

Condition (A.1) ensures that the functions at the righthand sides of (6) are locally Lipschitzian, which yields unique solutions to initial values.

\section{A.2. The proof of the Theorem 1 on the boundedness and invariance of solutions}

Proof. Let us prove by contradiction, i.e. assume that there is time $t^{*}>0$, s.t. a trajectory with initial conditions in $\Delta$ touches or crosses the boundary $\partial \Delta$ for the first time. Since the boundary consists of at most five surfaces (four if $K>P_{T} / q$ ), we consider five possible cases of crossing the boundary: $x\left(t^{*}\right)=0, x\left(t^{*}\right)=$ $k=\min \left(K, P_{T} / q\right), y_{1}\left(t^{*}\right)=0, y_{2}\left(t^{*}\right)=0$ and $q x\left(t^{*}\right)+$ $s_{1} y_{1}\left(t^{*}\right)+s_{2} y_{2}\left(t^{*}\right)=P_{T}$.

Case 1: Assume $x\left(t^{*}\right)=0$ (here we exclude the edge $P_{T}=s_{1} y_{1}-s_{2} y_{2}$ lying on $x=0$ plane). Let $P_{\min }=$ $\min _{t \in\left[0, t^{*}\right]}\left(P_{T}-s_{1} y_{1}(t)-s_{2} y_{2}(t)\right)>0, \quad$ also let $\tilde{y}_{i}=$ $\max _{t \in\left[0, t^{*}\right]} y_{i}(t)$ and note that $f_{i}(x) \leqslant f_{i}^{\prime}(0) x$. Then,

$$
\begin{aligned}
x^{\prime}(t)= & r x(t)\left(1-\frac{x(t)}{\min \left(K,\left(P_{T}-s_{1} y_{1}(t)-s_{2} y_{2}(t)\right) / q\right)}\right) \\
& -\sum_{i=1}^{2} f_{i}(x(t)) y_{i}(t) \\
\geqslant & \left(r\left(1-\frac{x(t)}{\min \left(K, P_{\min }\right)}\right)-\sum_{i=1}^{2} f_{i}^{\prime}(0) \tilde{y}_{i}\right) x(t) \equiv \alpha x(t),
\end{aligned}
$$


where $\alpha$ is some constant. Thus, $x(t) \geqslant x(0) e^{\alpha t}>0$, which implies that $x\left(t^{*}\right)>0$.

Case 2: Assume $x\left(t^{*}\right)=k$. Then

$x^{\prime}(t) \leqslant r x(t)\left(1-\frac{x(t)}{\min \left(K, \frac{P_{T}}{q}\right)}\right) \leqslant r x(t)\left(1-\frac{x(t)}{k}\right)$.

The standard comparison argument yields that $x(t)<k$ for all $t \geqslant 0$.

Cases 3 and 4: Assume that $y_{1}\left(t^{*}\right)=0$ or $y_{2}\left(t^{*}\right)=0$. Since $y_{i}^{\prime}(t) \geqslant-d_{i} y_{i}(t)$ it follows that $y_{i}(t) \geqslant y_{i}(0) e^{-d t}>0$, $i=1,2$ for $t \geqslant 0$.

Case 5 (includes the edge excluded in case 1): Assume

$q x\left(t^{*}\right)+s_{1} y_{1}\left(t^{*}\right)+s_{2} y_{2}\left(t^{*}\right)=P_{T}$.

Since for all $t \in\left[0, t^{*}\right), q x(t)+s_{1} y_{1}(t)+s_{2} y_{2}(t)<P_{T}$, it follows that

$\left.q x^{\prime}\left(t^{*}\right)+s_{1} y_{1}^{\prime}\left(t^{*}\right)+s_{2} y_{2}^{\prime} t^{*}\right) \geqslant 0$.

First, let us show that $x\left(t^{*}\right) \neq 0$ (to avoid division by 0 later). If $x\left(t^{*}\right)=0$, then $s_{1} y_{1}\left(t^{*}\right)+s_{2} y_{2}\left(t^{*}\right)=P_{T}$.

However,

$$
\begin{aligned}
\left(s_{1} y_{1}+s_{2} y_{2}\right)^{\prime} & \leqslant \sum_{i=1}^{2}\left(e_{i}\left(P_{T}-s_{1} y_{1}-s_{2} y_{2}\right) \frac{f_{i}(x)}{x} y_{i}-d_{i} y_{i}\right) \\
& \leqslant \sum_{i=1}^{2}\left(e_{i}\left(P_{T}-s_{1} y_{1}+s_{2} y_{2}\right) f_{i}^{\prime}(0) y_{i}\right) \\
& =\sum_{i=1}^{2}\left(e_{i} f_{i}^{\prime}(0) y_{i}\right) P_{T}\left(1-\frac{s_{1} y_{1}+s_{2} y_{2}}{P_{T}}\right),
\end{aligned}
$$

and the standard comparison argument yields that $s_{1} y_{1}(y)+s_{2} y_{2}(t)<P_{T}$ for all $t \geqslant 0$. Hence, $x\left(t^{*}\right) \neq 0$.

Observe that at $t^{*}$, the producer reaches its carrying capacity determined by available $\mathrm{P}$ and has the lowest quality, $q$, because (A.2) implies that

$$
\left(P_{T}-s_{1} y_{1}\left(t^{*}\right)-s_{2} y_{2}\left(t^{*}\right)\right) / q=x\left(t^{*}\right) .
$$

Substituting it to (6a), yields:

$$
\begin{aligned}
x^{\prime}\left(t^{*}\right) & =r x\left(t^{*}\right)\left(1-\frac{x\left(t^{*}\right)}{\min \left(K, x\left(t^{*}\right)\right)}\right)-\sum_{i=1}^{2} f_{i}\left(x\left(t^{*}\right)\right) y_{i}\left(t^{*}\right) \\
& \leqslant-\sum_{i=1}^{2} f_{i}\left(x\left(t^{*}\right)\right) y_{i}\left(t^{*}\right) .
\end{aligned}
$$

From (A.2) it also follows that $\left(P_{T}-s_{1} y_{1}\left(t^{*}\right)-\right.$ $\left.s_{2} y_{2}\left(t^{*}\right)\right) / x\left(t^{*}\right)=q$. Substituting it to (6b), (6c), yields bounds on $y_{i}^{\prime}\left(t^{*}\right), i=1,2$ :

$$
\begin{aligned}
y_{i}^{\prime}\left(t^{*}\right) & \leqslant e_{i} \min \left\{1, \frac{q}{s_{i}}\right\} f_{i}\left(x\left(t^{*}\right)\right) y_{i}\left(t^{*}\right) \\
& \leqslant e_{i} \frac{q}{s_{i}} f_{i}\left(x\left(t^{*}\right)\right) y_{i}\left(t^{*}\right) .
\end{aligned}
$$

Using (A.4), (A.5) and the fact that $e_{i}<1$, we obtain the following:

$$
\begin{aligned}
& q x^{\prime}\left(t^{*}\right)+s_{1} y_{1}^{\prime}\left(t^{*}\right)+s_{2} y_{2}^{\prime}\left(t^{*}\right) \\
& \quad \leqslant \sum_{i=1}^{2}\left(-1+e_{i}\right) q f_{i}\left(x\left(t^{*}\right)\right) y_{i}\left(t^{*}\right)<0 .
\end{aligned}
$$

This contradicts (A.3) and completes the proof.

\section{A.3. Jacobian, ecosystem matrix and equilibria}

If the following system has a solution

$F\left(x, y_{1}, y_{2}\right)=G_{1}\left(x, y_{1}, y_{2}\right)=G_{2}\left(x, y_{1}, y_{2}\right)=0$,

then system (10) has an internal (positive) equilibrium, which we denote as $\left(x^{*}, y_{1}^{*}, y_{2}^{*}\right)$.

To determine the type of species interactions occurring at this equilibrium, it is convenient to examine the Jacobian of system (10):

$$
\begin{aligned}
& J\left(x, y_{1}, y_{2}\right) \equiv\left(a_{i j}\right)_{3 \times 3} \\
& \equiv\left(\begin{array}{ccc}
F+\frac{\partial F}{\partial x} x & \frac{\partial F}{\partial y_{1}} x & \frac{\partial F}{\partial y_{2}} x \\
\frac{\partial G_{1}}{\partial x} y_{1} & G_{1}+\frac{\partial G_{1}}{\partial y_{1}} y_{1} & \frac{\partial G_{1}}{\partial y_{2}} y_{1} \\
\frac{\partial G_{2}}{\partial x} y_{2} & \frac{\partial G_{2}}{\partial y_{1}} y_{2} & G_{2}+\frac{\partial G_{2}}{\partial y_{2}} y_{2}
\end{array}\right) .
\end{aligned}
$$

Then the Jacobian at $\left(x^{*}, y_{1}^{*}, y_{2}^{*}\right)$ is

$J\left(x^{*}, y_{1}^{*}, y_{2}^{*}\right)=\left(\begin{array}{ccc}x^{*} & 0 & 0 \\ 0 & y_{1}^{*} & 0 \\ 0 & 0 & y_{2}^{*}\end{array}\right) E\left(x^{*}, y_{1}^{*}, y_{2}^{*}\right)$,

where

$$
E\left(x^{*}, y_{1}^{*}, y_{2}^{*}\right)=\left(\begin{array}{lll}
\frac{\partial F}{\partial x} & \frac{\partial F}{\partial y_{1}} & \frac{\partial F}{\partial y_{2}} \\
\frac{\partial G_{1}}{\partial x} & \frac{\partial G_{1}}{\partial y_{1}} & \frac{\partial G_{1}}{\partial y_{2}} \\
\frac{\partial G_{2}}{\partial x} & \frac{\partial G_{2}}{\partial y_{1}} & \frac{\partial G_{2}}{\partial y_{2}}
\end{array}\right)
$$

is the ecosystem matrix of our system. In this matrix, the $i j$ th term measures the effect of the $j$ th species on the $i$ th species growth rate.

If prey quality is good for both consumers, i.e. (17) holds, then, by substituting signs in (13)-(15) into (A.9), we obtain (18).

If prey quality is bad for both consumers, i.e. (16) holds, then by substituting signs given by (13)-(15) into (A.9) we obtain (19).

In the case of bad prey quality,

$$
A \equiv P_{T}-s_{1} y_{1}^{*}-s_{2} y_{2}^{*}=\frac{d_{1} s_{1} x^{*}}{e_{1} f_{1}\left(x^{*}\right)}=\frac{d_{2} s_{2} x^{*}}{e_{2} f_{2}\left(x^{*}\right)}
$$


and

$$
\begin{aligned}
B & \equiv r x^{*}\left(1-\frac{x^{*}}{\min \left(K,\left(d_{2} s_{2} x^{*}\right) /\left(q e_{2} f_{2}\left(x^{*}\right)\right)\right.}\right) \\
& =f_{1}\left(x^{*}\right) y_{1}^{*}+f_{2}\left(x^{*}\right) y_{2}^{*} .
\end{aligned}
$$

Then the value of $x^{*}$ is determined by

$\frac{d_{1} s_{1} x^{*}}{e_{1} f_{1}\left(x^{*}\right)}=\frac{d_{2} s_{2} x^{*}}{e_{2} f_{2}\left(x^{*}\right)}$,

and the values of $y_{1}^{*}$ and $y_{2}^{*}$ are given by

$$
\begin{aligned}
& y_{1}^{*}=\frac{f_{2}\left(x^{*}\right) P_{T}-s_{2} B-f_{2}\left(x^{*}\right) A}{s_{1} f_{2}\left(x^{*}\right)-s_{2} f_{1}\left(x^{*}\right)}, \\
& y_{2}^{*}=\frac{f_{1}\left(x^{*}\right) A+s_{1} B-f_{1}\left(x^{*}\right) P_{T}}{s_{1} f_{2}\left(x^{*}\right)-s_{2} f_{1}\left(x^{*}\right)} .
\end{aligned}
$$

Since (A.10) does not depend on $K$, increasing $K$ does not effect $x^{*}$. Because $B$ can increase with $K$, increasing $K$ can change $y_{1}^{*}$ and $y_{2}^{*}$ in opposite directions (Figs. 1 and 3 illustrate this case).

Observe that model (6) has $E_{0} \equiv(0,0,0)$ and $E_{k} \equiv$ $\left(\min \left(K, P_{T} / q\right), 0,0\right)$ as its only axial equilibria. Clearly, $E_{0}$ is always a saddle. It is easy to show that if per capita growth rate of each consumer is negative at $E_{k}$, i.e. if $G_{i}\left(E_{k}\right)<0, i=1,2$, then $E_{k}$ is locally asymptotically stable and no consumer can invade the system around this equilibrium. For general functions $f_{i}(x)$ satisfying (2), there can be several other nonnegative equilibria on the boundary surfaces $\left(x-y_{i}\right.$ surface, $\left.i=1,2\right)$ of $\Delta$ (Loladze et al., 2000). The stability of these equilibria and positive equilibria (if any) can be routinely studied via Routh-Hurwitz criteria (Edelstein-Keshet, 1988, p. 234) when specific functions $f_{i}(x), i=1,2$ are given. Persistent properties of the system can be obtained by applying existing theory (e.g. Thieme, 1993, and the references therein).

\section{References}

Abrams, P.A., 1988. How should resources be counted. Theor. Popul. Biol. 33, 226-242.

Abrams, P.A., Holt, R.D., 2002. The impact of consumer-resource cycles on the coexistence of competing consumers. Theor. Popul. Biol. 62, 281-295.

Adams, R.S., 1974. Variability in mineral and trace-element content of dairy-cattle feeds. J. Dairy Sci. 58, 1538-1548.

Andersen, T., 1997. Pelagic Nutrient Cycles: Herbivores as Sources and Sinks. Springer, New York, NY.

Andersen, T., Hessen, D.O., 1991. Carbon, nitrogen, and phosphorus content of freshwater zooplankton. Limnol. Oceanogr. 36, 807-814.

Armstrong, R.A., McGehee, R., 1976. Coexistence of 2 competitors on one resource. J. Theor. Biol. 56, 499-502.

Armstrong, R.A., McGehee, R., 1980. Competitive exclusion. Am. Nat. 115, 151-170.

Butler, G.J., Hsu, S.B., Waltman, P., 1985. A mathematical-model of the chemostat with periodic washout rate. SIAM J. Appl. Math. $45,435-449$.
Chase, J.M., Abrams, P.A., Grover, J.P., Diehl, S., Chesson, P., Holt, R.D., Richards, S.A., Nisbet, R.M., Case, T.J., 2002. The interaction between predation and competition: a review and synthesis. Ecol. Lett. 5, 302-315.

Chesson, P., 1994. Multispecies competition in variable environments. Theor. Popul. Biol. 45, 227-276.

Chesson, P., 2000. Mechanisms of maintenance of species diversity. Annu. Rev. Ecol. Syst. 31, 343-366.

Codeco, C.T., Grover, J.P., 2001. Competition along a spatial gradient of resource supply: a microbial experimental model. Am. Nat. 157, 300-315.

Cotrufo, M.F., Ineson, P., Scott, A., 1998. Elevated CO2 reduces the nitrogen concentration of plant tissues. Glob. Change Biol. 4, 43-54.

Daufresne, T., Loreau, M., 2001a. Plant-herbivore interactions and ecological stoichiometry: when do herbivores determine plant nutrient limitation? Ecol. Lett. 4, 196-206.

Daufresne, T., Loreau, M., 2001b. Ecological stoichiometry, primary producer-decomposer interactions, and ecosystem persistence. Ecology 82, 3069-3082.

DeMott, W.R., 1998. Utilization of cyanobacterium and phosphorusdeficient green alga as a complementary resources by daphnids. Ecology 79, 2463-2481.

Diehl, S., 2002. Phytoplankton, light, and nutrients in a gradient of mixing depths: Theory. Ecology 83, 386-398.

Diehl, S., 2003. The evolution and maintenance of omnivory: dynamic constraints and the role of food quality. Ecology, in press.

Droop, M.R., 1974. The nutrient status of algal cells in continuous culture. J. Mar. Biol. Assoc. UK 55, 825-855.

Durrett, R., Levin, S.A., 1994. The importance of being discrete (and spatial). Theor. Pop. Biol. 46, 363-394.

Edelstein-Keshet, L., 1988. Mathematical Models in Biology. Random House, New York.

Elser, J.J., Urabe, J., 1999. The stoichiometry of consumer-driven nutrient recycling: theory, observations, and consequences. Ecology $80,735-751$.

Elser, J.J., Dobberfuhl, D., MacKay, N.A., Schampel, J.H., 1996. Organism size, life history, and N:P stoichiometry: towards a unified view of cellular and ecosystem processes. BioScience 46, 674-684.

Elser, J.J., Sterner, R.W., Gorokhova, E., Fagan, W.F., Markow, T.A., Cotner, J.B., Harrison, J.F., Hobbie, S.E., Odell, G.M., Weider, L.J., 2000. Biological stoichiometry from genes to ecosystems. Ecol. Lett. 3, 540-550.

Fagan, W.F., Bishop, J.G., 2000. Trophic interactions during primary succession: herbivores slow a plant reinvasion at Mount St. Helens. Am. Nat. 155, 238-251.

Fryxell, J.M., 1991. Forage quality and aggregation by large herbivores. Am. Nat. 138, 478-498.

Gause, G.F., 1934. The Struggle for Existence. Williams \& Wilkins, Baltimore, MD (reprinted in 1964 and 1969 by Hafner, NY and in 1971 by Dover, New York).

Grover, J.P., 1997. Resource Competition. Chapman \& Hall, London, UK.

Grover, J.P., 2002. Stoichiometry, herbivory and competition for nutrients: Simple models based on planktonic ecosystems. J. Theor. Biol. 214, 599-618.

Hardin, G., 1959. Nature and Man's Fate. Rinehart, New York.

Hardin, G., 1960. Competitive exclusion principle. Science 131, 1292-1297.

Hastings, A., 1980. Disturbance, coexistence, history and competition for space. Theor. Popul. Biol. 18, 363-373.

Holt, R.D., Lawton, J.H., 1994. The ecological consequences of shared natural enemies. Annu. Rev. Ecol. Syst. 25, 495-520.

Hsu, S.B., Cheng, K.S., Hubbell, S.P., 1981. Exploitative competition of microorganism for two complementary nutrients in continuous culture. SIAM J. Appl. Math. 41, 422-444. 
Huisman, J., Weissing, F.J., 1994. Light-limited growth and competition for light in well-mixed aquatic environments: an elementary model. Ecology 75, 507-520.

Huisman, J., Weissing, F.J., 1999. Biodiversity of plankton by species oscillations and chaos. Nature 402, 407-410.

Huisman, J., Weissing, F.J., 2000. Coexistence and resource competition. Nature 407, 694

Jablonski, L.M., Wang, X.Z., Curtis, P.S., 2002. Plant reproduction under elevated $\mathrm{CO} 2$ conditions: a meta-analysis of reports on 79 crop and wild species. New Phytol. 156, 9-26.

Kuang, Y., Fagan, W.F., Loladze, I., 2003. Biodiversity, habitat area, resource growth rate and interference competition. Bull. Math. Biol. 65, 497-518.

Leibold, M.A., 1996. A graphical model of keystone predators in food webs: trophic regulation of abundance, incidence, and diversity patterns in communities. Am. Nat. 147, 784-812.

Lenas, P., Pavlou, S., 1995. Coexistence of 3 competing microbial populations in a chemostat with periodically varying dilution rate. Math. Biosci. 129, 111-142.

Leon, J.A., Tumpson, D.B., 1975. Competition between two species for two complementary or substitutable resources. J. Theor. Biol. $50,185-201$

Levin, S.A., 1970. Community equilibria and stability, and an extension of the competitive exclusion principle. Am. Nat. 104, 413-423.

Li, B.T., Wolkowicz, G.S.K., Kuang, Y., 2000. Global asymptotic behavior of a chemostat model with two perfectly complementary resources and distributed delay. SIAM J. Appl. Math. 60, 2058-2086.

Litchman, E., Klausmeier, C.A., 2001. Competition of phytoplankton under fluctuating light. Am. Nat. 157, 170-187.

Loladze, I., 2002. Rising atmospheric CO2 and human nutrition: toward globally imbalanced plant stoichiometry? Trends Ecol. Evol. 17, 457-461

Loladze, I., Kuang, Y., Elser, J.J., 2000. Stoichiometry in producergrazer systems: Linking energy flow with element cycling. Bull. Math. Biol. 62, 1137-1162.

Lotka, A.J., 1925. Elements of Physical Biology. Williams and Wilkins, Baltimore (Reprinted as Elements of Mathematical Biology (1956) Dover, NY).

MacArthur, R., Levins, R., 1964. Competition, habitat selection, and character displacement in a patchy environment. Proc. Natl. Acad. Sci. USA 51, 1207-1210.

Main, T.M., Dobberfuhl, D.R., Elser, J.J., 1997. N:P stoichiometry and ontogeny of crustacean zooplankton: a test of the growth rate hypothesis. Limnol. Oceanogr. 42, 1474-1478.

McCann, K., Hastings, A., Huxel, G.R., 1998. Weak trophic interactions and the balance of nature. Nature 395, 794-798.

McCann, K.S., 2000. The diversity-stability debate. Nature 405, 228-233.
Milewski, A.V., Diamond, R.E., 2000. Why are very large herbivores absent from Australia? A new theory of micronutrients. J. Biogeography 27, 957-978.

Muller, E.B., Nisbet, R.M., Kooijman, S.A.L.M., Elser, J.J., McCauley, E., 2001. Stoichiometric food quality and herbivore dynamics. Ecol. Lett. 4, 519-529.

Nelson, W.A., McCauley, E., Wrona, F.J., 2001. Multiple dynamics in a single predator-prey system: experimental effects of food quality. Proc. R. Soc. Lond. [Biol]. 268, 1223-1230.

Richards, S.A., Nisbet, R.M., Wilson, W.G., Possingham, H.P., 2000. Grazers and diggers: exploitation competition and coexistence among foragers with different feeding strategies on a single resource. Am. Nat. 155, 266-279.

Rosenzweig, M.L., 1971. Paradox of enrichment: destabilization of exploitation ecosystems in ecological time. Science 171, 385-387.

Smith, H.L., Waltman, P., 1995. The Theory of the Chemostat. Cambridge University Press, Cambridge.

Sterner, R.W., Elser, J.J., 2002. Ecological Stoichiometry. Princeton University, Princeton, NJ.

Sterner, R.W., Hessen, D.O., 1994. Algal nutrient limitation and the nutrient of aquatic herbivores. Ann. Rev. Ecol. Syst. 25, 1-29.

Sterner, R.W., Clasen, J., Lampert, W., Weisse, T., 1998. Carbon: phosphorus stoichiometry and food chain production. Ecol. Lett. $1,146-150$.

Strobeck, C., 1973. N species competition. Ecology 54, 650-654.

Thieme, H.R., 1993. Persistence under relaxed point-dissipativity (with applications to an endemic model). SIAM J. Math. Anal. 24, 407-435.

Tilman, D., 1982. Resource Competition and Community Structure. Princeton University Press, Princeton.

Urabe, J., Sterner, R.W., 1996. Regulation of herbivore growth by the balance of light and nutrients. Proc. Natl. Acad. Sci. USA 93, 8465-8469.

Urabe, J., Elser, J.J., Kyle, M., Yoshida, T., Sekino, T., Kawabata, Z., 2002. Herbivorous animals can mitigate unfavourable ratios of energy and material supplies by enhancing nutrient recycling. Ecol. Lett. 5, 177-185.

Urabe, J., Togari, J., Elser, J.J., 2003. Stoichiometric impacts of increased carbon dioxide on planktonic herbivores. Glob. Change Biol. 9, 818-825.

Vance, R.R., 1985. The stable coexistence of two competitors for one resource. Am. Nat. 126, 72-86.

Vance, R.R., 1984. Interference competition and the coexistence of two competitors on a single limiting resource. Ecology 65, 1349-1357.

Volterra, V., 1926. Fluctuations in the abundance of a species considered mathematically. Nature 118, 558-560.

Williams, R.J.P., Frausto da Silva, J.J.R., 2001. The Biological Chemistry of the Elements: The Inorganic Chemistry of Life, 2nd Edition. Oxford University Press, New York. 